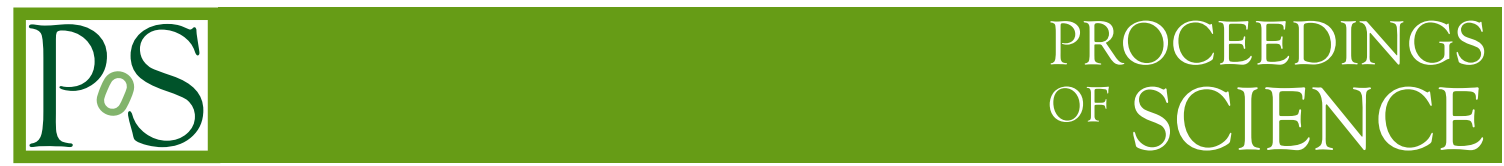

\title{
Searching for supersymmetric partners of top quarks at the CMS experiment
}

\section{Soham Bhattacharya ${ }^{a}$ on behalf of the CMS collaboration}

${ }^{a}$ Tata Institute of Fundamental Research, Mumbai, India

E-mail: soham.bhattacharya@cern.ch

\begin{abstract}
The newest results for classic searches for the pair-production of supersymmetric top partners in final states with and without leptons with the CMS detector are presented. The analysis strategies are developed to cover different scenarios of heavy top squarks as well as those with top squark mass close to the Standard Model top quarks. The results are based on proton-proton collisions recorded at $\sqrt{s}=13 \mathrm{TeV}$ with the CMS detector using the full Run 2 dataset of $137 \mathrm{fb}^{-1}$.
\end{abstract}

40th International Conference on High Energy physics - ICHEP2020

July 28 - August 6, 2020

Prague, Czech Republic (virtual meeting) 


\section{Introduction}

The Standard Model (SM) is the most successful theory of elementary particle physics till date. However, it is an incomplete theory as it is unable to provide satisfactory answers to various fundamental problems like the existence of dark matter/energy, origin of gravitational interaction, stability of the Higgs boson mass, etc. Supersymmetry (SUSY) is one of the most widely studied beyond standard model (BSM) theories that have been proposed to explain the shortcomings of the Standard Model. SUSY postulates that for every boson there exists a fermionic superpartner and vice versa. The bosonic superpartner of the top quark, the top squark, plays an important role in stabilizing the Higgs boson mass by canceling the dominant top quark loop correction. The SUSY partners of left- and right-handed top quarks are the top squarks, $\tilde{t}_{L}$ and $\tilde{t}_{R}$ respectively. These particles can mix with each other, resulting in physical states $\tilde{t}_{1}$ and $\tilde{t}_{2}$, with $\tilde{t}_{1}$ defined to be the lighter of the two. Hence searches for top squarks exploring a wide variety of SUSY models, have received a lot of attention at the Compact Muon Solenoid (CMS) experiment. The typical benchmark scenarios exploring different regions of the SUSY parameter space are illustrated in Fig. 1. Depending on the mass hierarchy and the composition (bino, wino, or higgsino like) of the charginos and neutralinos, each of these scenarios will have different final state particles and kinematic properties, thereby requiring a dedicated search that is optimized to be sensitive to a particular event topology.

The CMS experiment recorded $137 \mathrm{fb}^{-1}$ of high quality proton-proton collision data at a center of mass energy of $13 \mathrm{TeV}$, during its Run 2 data taking period. This report presents the following studies with the full Run 2 dataset. The searches for $\tilde{t}_{1}$ pair production in a final state containing two leptons [1] or a single lepton and jets [2] are presented in Sec. 2; and Sec. 3 describes the search for $\tilde{t}_{2}$ pair production in a final state containing jets and two same-sign leptons or multiple leptons [3]. These searches have been carried out within the simplified model spectra (SMS) framework [4, 5], and they probe the type (a) and type (b) scenarios presented in Fig. 1.

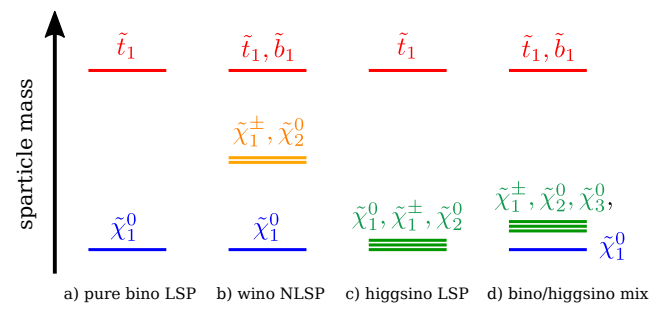

Figure 1: A schematic diagram showing the typical benchmark scenarios for top squark searches.

\section{Search for $\tilde{t}_{1}$ pair production}

\subsection{Dilepton final state}

This search [1] considers the strong production of $\tilde{t}_{1}$ pairs, where the $\tilde{t}_{1}$ decays in one of the following modes: $\tilde{t}_{1} \rightarrow t \tilde{\chi}_{1}^{0}, \tilde{t}_{1} \rightarrow b \tilde{\chi}_{1}^{+} \rightarrow b W^{+} \tilde{\chi}_{1}^{0}$ or $\tilde{t}_{1} \rightarrow b \tilde{\chi}_{1}^{+} \rightarrow b \tilde{\ell}^{+} v \rightarrow b \ell^{+} \tilde{\chi}_{1}^{0} v$. The chargino $\left(\tilde{\chi}_{1}^{ \pm}\right)$ mass is parameterized as $m_{\tilde{\chi}_{1}^{ \pm}}=0.5\left(m_{\tilde{t}_{1}}+m_{\tilde{\chi}_{1}^{0}}\right)$, and the slepton $(\tilde{\ell})$ mass as, $m_{\tilde{\ell}}=m_{\tilde{\chi}_{1}^{0}}+x\left(m_{\tilde{\chi}_{1}^{ \pm}}+m_{\tilde{\chi}_{1}^{0}}\right)$, where $x=[0.05,0.5,0.95]$. Only the leptonic decay modes of the top quarks and $W$ bosons have been considered for this study, and the decays are assumed to be identical for all three lepton flavors. 

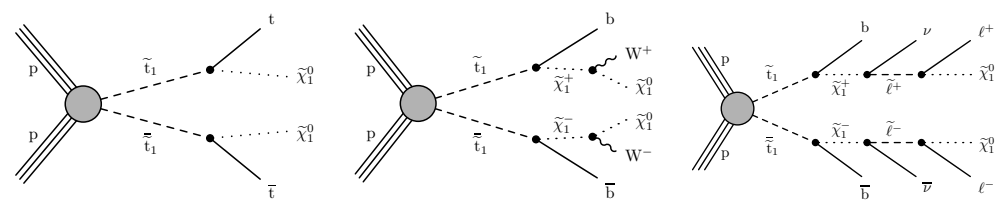

Figure 2: The diagrams corresponding to the SMS framework models that have been considered in the search detailed in Sec. 2.1.

Events in the signal region are selected with single lepton or dilepton triggers, and required to satisfy the following criteria:

- At least two oppositely charged leptons.

- The invariant mass of the two leptons must satisfy the conditions $m_{\ell \ell}>20 \mathrm{GeV}$ and $\left|m_{\ell \ell}-m_{Z}\right|>$ $15 \mathrm{GeV}$.

- At least two jets, and at least one $b$-tagged jet.

- The angle between the missing transverse momentum $\left(p_{T}^{\text {miss }}\right)$ and the jets with the highest $\left(j_{1}\right)$ and second highest $\left(j_{2}\right)$ transverse momenta must satisfy the conditions $\cos \Delta \phi\left(p_{T}^{\text {miss }}, j_{1}\right)<0.8$ and $\cos \Delta \phi\left(p_{T}^{\text {miss }}, j_{2}\right)<0.96$.

- The $p_{T}^{\text {miss }}$ significance $(\mathcal{S})$ must satisfy $\mathcal{S}>12$.

The phase space selected with the aforementioned criteria is subdivided into multiple bins in terms of $\mathcal{S}$ and the "stransverse masses" $m_{T 2}(\ell \ell)$ and $m_{T 2}(b \ell b \ell)$. The dominant source of background with genuine leptons is from $t \bar{t}$ events; a normalization correction factor is extracted from a signal depleted control region $\left(m_{T 2}(\ell \ell)<100 \mathrm{GeV}\right)$ to correct the prediction from simulation. The tails of the $m_{T 2}$ distributions can be populated due to effects such as jet momentum resolution, mismeasurement of jet momentum, electrons/muons failing the identification criteria, or tau lepton decays. In order to ensure that these effects are well modeled in the background prediction, the data are compared to the prediction in different control regions (CRs). Correction factors for the subdominant background processes (Drell-Yan, multiboson, etc.) are derived from fits in CRs. No significant deviation with respect to the SM prediction was observed, and the exclusion limits on the top squark mass in terms of the lightest neutralino mass are presented in Fig. 3 for the three different top squark decay modes discussed above.
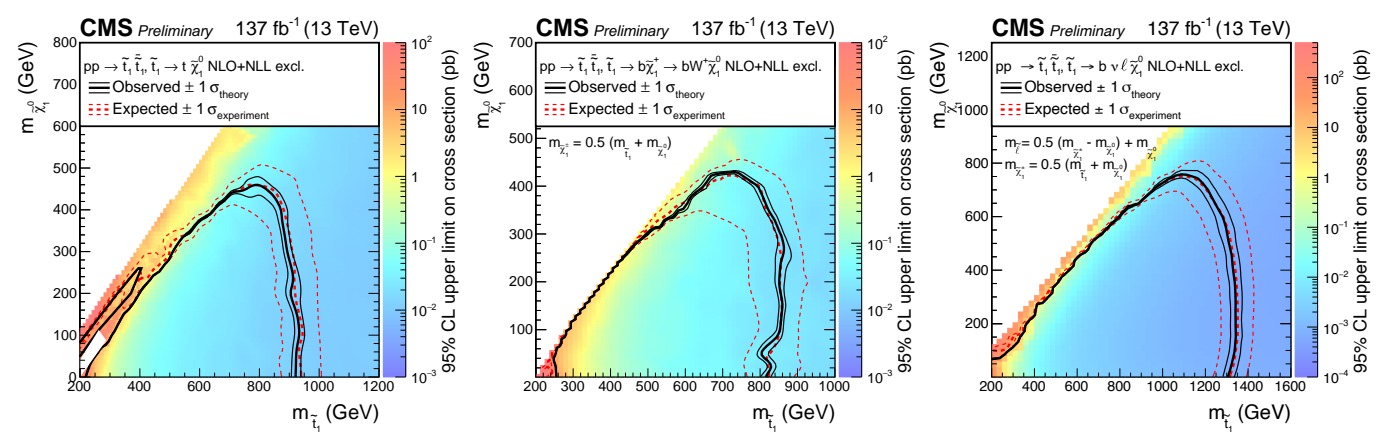

Figure 3: Exclusion limits [1] in the $m_{\tilde{t}_{1}}$ and $m_{\tilde{\chi}_{1}^{0}}$ plane for the top squark decay modes considered in the search described in Sec. 2.1. 


\subsection{Final state with one lepton and jets}

This search [2] probes top squark pair production models in which the top squark decays as $\tilde{t}_{1} \rightarrow t \tilde{\chi}_{1}^{0}$, or $\tilde{t}_{1} \rightarrow b \tilde{\chi}_{1}^{+} \rightarrow b W^{+} \tilde{\chi}_{1}^{0}$. The SMS model diagrams are presented in Fig. 4. The chargino mass parameterization is the same as that in the previous search. This study considers the situation in which one top quark (or $W$ boson) decays leptonically and the other hadronically, thereby producing a final state containing one lepton and jets.
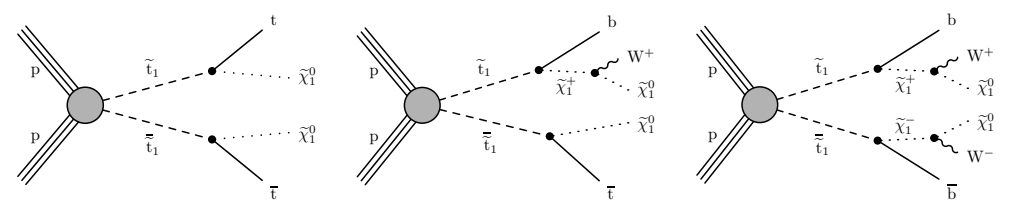

Figure 4: The diagrams corresponding to the SMS framework models that have been considered in the search detailed in Sec. 2.2.

Events are selected with $p_{T}^{\text {miss }}, H_{T}$ (scalar sum of jet transverse momenta) and single lepton triggers, and required to satisfy the following criteria:

- Exactly one isolated lepton.

- At least two jets $\left(N_{j} \geq 2\right)$, and at least one $b$-tagged jet.

- $p_{T}^{\text {miss }}>250 \mathrm{GeV}$.

- The transverse mass between the lepton and $p_{T}^{\text {miss }}$ satisfies the condition $m_{T}\left(\ell, p_{T}^{\text {miss }}\right)>150 \mathrm{GeV}$. - $\min \left[\Delta \phi\left(p_{T}^{\text {miss }}, j_{1}\right), \Delta \phi\left(p_{T}^{\text {miss }}, j_{2}\right)\right]>0.8$ or $>0.5$ for the compressed scenario $\left(\Delta m\left(\tilde{t}_{1}-\tilde{\chi}_{1}^{0}\right) \sim m_{t}\right.$ or $\left.\Delta m\left(\tilde{t}_{1}-\tilde{\chi}_{1}^{0}\right) \sim m_{W}\right)$.

A deep neural network (DNN) is used to tag the hadronically decaying top quark in one of the three categories: untagged, resolved, or merged. In addition to the standard $b$-tagging algorithm, this study also employs a soft $(>1 \mathrm{GeV}) b$-tagging technique that uses secondary vertices which are not associated to any jet or lepton. This is found to improve the sensitivity in the compressed region mentioned above. The search region thus selected is divided into 39 bins in terms of $N_{j}$, "topness" $t_{\text {mod }}$, invariant mass between the lepton and the $b$-tagged jet $\left(m_{\ell b}\right), p_{T}^{\text {miss }}$, and the toptagging category. The $t_{\text {mod }}$ variable is a $\chi^{2}$-like variable that helps to discriminate between signal (high $t_{\text {mod }}$ ) and leptonically decaying $t \bar{t}$ (low $t_{\text {mod }}$ ) events.

The major background is from "lost lepton" processes, which are events with two leptonically decaying $W$ bosons where one of the leptons is not reconstructed or fails the identification criteria. The contribution from such events is estimated in a data-driven manner, using a dilepton CR in data. The contribution from genuine single lepton events is estimated from a CR in data containing no $b$-tagged jets. Lastly, events with a leptonically decaying $W$ boson and a $Z$ boson decaying to neutrinos, are estimated from simulation. No significant deviation with respect to the SM prediction was observed, and the exclusion limits on the top squark mass in terms of the lightest neutralino mass are presented in Fig. 5 for the three top squark different decay modes discussed is this section.

\section{Search for $\tilde{t}_{2}$ pair production in a final state with jets and two same-sign leptons or multiple leptons}

The study [3] presented here searches for pair production of the heavier top squark $\left(\tilde{t}_{2}\right)$, where $\tilde{t}_{2}$ decays to $\tilde{t}_{1}$ and a $Z\left(\tilde{t}_{2} \rightarrow \tilde{t}_{1} Z\right)$ or Higgs boson $\left(\tilde{t}_{2} \rightarrow \tilde{t}_{1} H\right)$. Thus the signal contains two 

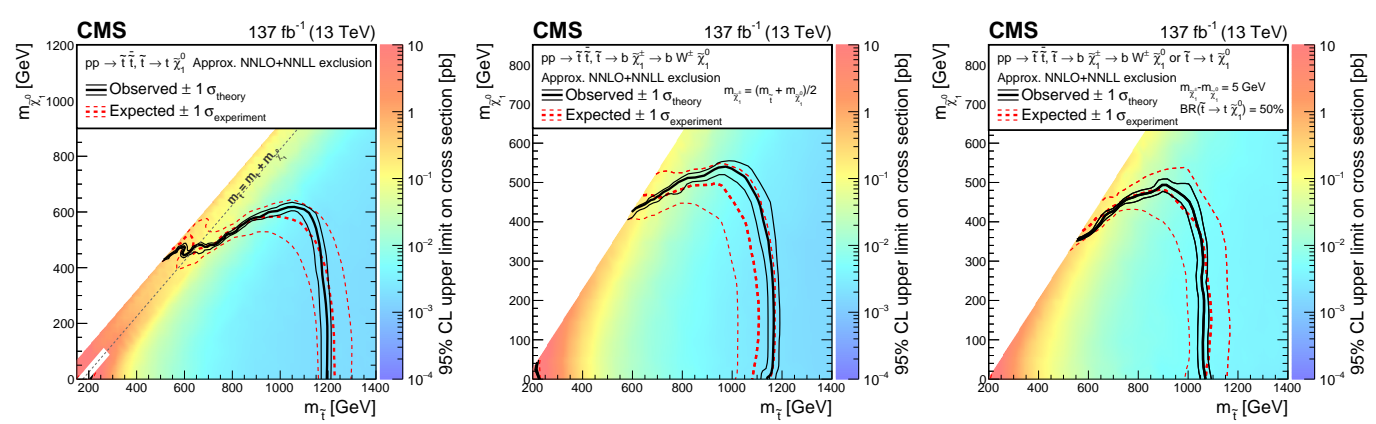

Figure 5: Exclusion limits [2] in the $m_{\tilde{t}_{1}}$ and $m_{\tilde{\chi}_{1}^{0}}$ plane for the top squark decay modes considered in the search described in Sec. 2.2.

Higgs bosons, or two $Z$ bosons, or one of each. The $\tilde{t}_{1}$ subsequently decays as $\tilde{t}_{1} \rightarrow t \tilde{\chi}_{1}^{0}$, and $m_{\tilde{t}_{1}}-m_{\tilde{\chi}_{1}^{0}}$ is considered to be $175 \mathrm{GeV}$, which is approximately equal to the top quark mass. Three different values of the branching fraction $\mathcal{B}\left(\tilde{t}_{2} \rightarrow \tilde{t}_{1} Z\right)$ have been considered: $0 \%, 50 \%$ and $100 \%$. This region of the SUSY phase space is the most difficult one to target with traditional top squark searches, and hence it helps to search for cascade decays in order to constrain such models.

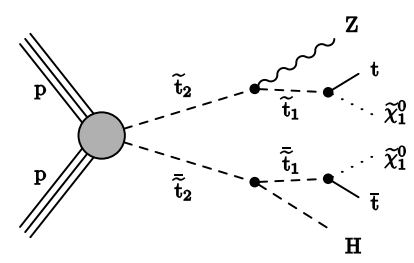

Figure 6: The diagram corresponding to the SMS framework model that has been considered in the search detailed in Sec. 3.

Events are selected with dilepton triggers, and required to satisfy the following criteria:

- At least two same-sign (SS) leptons.

- At least two jets $\left(N_{j} \geq 2\right)$.

- Reject if the lepton invariant mass satisfies $m_{\ell \ell}<12 \mathrm{GeV}$ for same flavor leptons.

- Reject if $m_{\ell \ell}<8 \mathrm{GeV}$ for leptons of any charge and flavor.

The search covers the phase space containing both high $(\mathrm{H}:>25 \mathrm{GeV})$ and low $(\mathrm{L}:<25 \mathrm{GeV}) p_{T}$ leptons and considers the following categories:

- HH, HL, LL: exactly two leptons and $p_{T}^{\text {miss }}>50 \mathrm{GeV}$.

- LM (low $p_{T}^{\text {miss }}$ ): exactly two leptons and $p_{T}^{\text {miss }}<50 \mathrm{GeV}$.

- ML (multilepton): $\geq 3$ leptons and $p_{T}^{\text {miss }}>50 \mathrm{GeV}$.

This strategy helps to improve the sensitivity in the compressed region where the leptons are soft. Each of these categories is subdivided in terms of $N_{j}, N_{b}, H_{T}$, the SS pair sign, $p_{T}^{\mathrm{miss}}$, and $m_{T}^{\mathrm{min}}$, which is the minimum of the transverse mass between $p_{T}^{\text {miss }}$ and each of the leptons forming the SS pair. The SM background with two or more prompt leptons (including an SS pair) is estimated from simulation with various correction factors applied. The contribution from events with at least one nonprompt lepton is estimated using a CR in data, by evaluating the probability of a nonprompt lepton being misidentified as prompt. The background also contains events with a pair of opposite- 
sign leptons, one of which has been reconstructed with the wrong charge. This contribution is estimated by parameterizing the lepton charge misidentification rate as a function its $p_{T}$ and $\eta$, which is found to be $10^{-5}-5 \times 10^{-3}$ for electrons and negligible for muons. No significant deviation with respect to the SM prediction was observed, and the exclusion limits on the heavier top squark mass in terms of the lighter one are presented in Fig. 7 for the three values of the branching fraction $\mathcal{B}\left(\tilde{t}_{2} \rightarrow \tilde{t}_{1} Z\right)$ stated above.
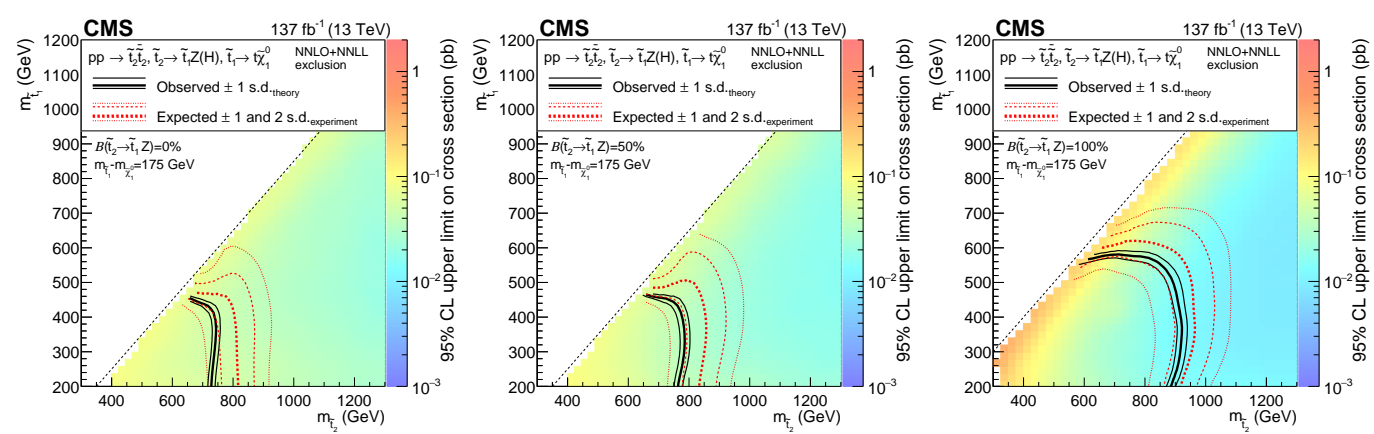

Figure 7: Exclusion limits [3] in the $m_{\tilde{t}_{2}}$ and $m_{\tilde{t}_{1}}$ plane for the branching fraction $\mathcal{B}\left(\tilde{t}_{2} \rightarrow \tilde{t}_{1} Z\right)$ values of $0 \%$ (left), $50 \%$ (center) and 100\% (right), as described in Sec. 3.

\section{Summary}

This report has presented the recent results of top squark searches at the CMS experiment, exploring different benchmark scenarios. Even though no signature of new physics has been discovered, these results help place constrains on different new physics models. Dedicated top squark searches targeting specific regions of the phase space (like the top mass corridor) are presently underway to ensure that all of the accessible parameter space is explored. In addition, the LHC is preparing for Run 3, which we hope will shed more light on the existence and nature of new physics.

\section{References}

[1] CMS collaboration, Search for top squark pair production using dilepton final states in pp collision data collected at $\sqrt{s}=13 \mathrm{TeV}, 2008.05936$.

[2] CMS collaboration, Search for direct top squark pair production in events with one lepton, jets, and missing transverse momentum at $13 \mathrm{TeV}$ with the CMS experiment, JHEP 05 (2020) 032 [1912. 08887].

[3] CMS collaboration, Search for physics beyond the standard model in events with jets and two same-sign or at least three charged leptons in proton-proton collisions at $\sqrt{s}=13 \mathrm{TeV}$, Eur. Phys. J. C 80 (2020) 752 [2001.10086].

[4] J. Alwall, P. Schuster and N. Toro, Simplified models for a first characterization of new physics at the LHC, Phys. Rev. D 79 (2009) 075020 [0810 . 3921].

[5] LHC New Physics Working Group collaboration, Simplified models for LHC new physics searches, J. Phys. G 39 (2012) 105005 [1105 . 2838]. 